\title{
Phylogenetic Relationships of Brown Frogs from Taiwan and Japan Assessed by Mitochondrial Cytochrome $b$ Gene Sequences (Rana: Ranidae)
}

\author{
Tomoko Tanaka-Ueno ${ }^{1 *}$, Masafumi Matsui ${ }^{1}$, Szu-Lung Chen², \\ Osamu Takenaka ${ }^{3}$ and Hidetoshi Ota ${ }^{4}$ \\ ${ }^{1}$ Graduate School of Human and Environmental Studies, Kyoto University, \\ Sakyo-ku, Kyoto 606-01, Japan \\ ${ }^{2}$ Department of Zoology, Graduate School of Science, Kyoto University, \\ Sakyo-ku, Kyoto 606-01, Japan \\ ${ }^{3}$ Primate Research Institute, Kyoto University, Inuyama, Aichi 484, Japan \\ ${ }^{4}$ Tropical Biosphere Research Center, University of the Ryukyus, \\ Nishihara, Okinawa 903-01, Japan
}

\begin{abstract}
In order to assess phylogenetic relationships of Taiwanese brown frogs (Rana longicrus and the $R$. sauteri complex), the partial sequences (587 base pairs) of the mitochondrial cytochrome $b$ genes were compared with six brown frogs from Japan $(R$. pirica, $R$. ornativentris, $R$. japonica, $R$. tagoi tagoi, $R$. tsushimensis, and $R$. okinavana). Resultant phylogenetic trees indicated a considerable genetic differentiation between $R$. longicrus and $R$. japonica in spite of their close morphological and ecological similarities. The $R$. sauteri complex includes two genetically distinct groups that are not consistent with current classification. One group including populations of Alishan (central Taiwan) and Sanyi (western Taiwan) seemed to be closest to $R$. tagoi and the presumptive common ancestor of these frogs is thought to have diverged very early. Another group including a population from Wulai (northern Taiwan) showed a sister relationship with $R$. tsushimensis and R. okinavana, both isolated on small islands of Japan. These Taiwanese and Japanese brown frogs as a whole form a monophyletic group, and separation of the $R$. sauteri complex as a distinct genus or subgenus Pseudorana was not supported.
\end{abstract}

\section{INTRODUCTION}

Taiwan is a subtropical island located $160 \mathrm{~km}$ east of the continental China. Being endowed with high mountains over $3500 \mathrm{~m}$, this large island accomodates diverse geographic and climatic environments and encompasses amphibian fauna consisting not only of the Oriental elements but also of the Palearctic ones as well (Zhao and Adler, 1993). The brown frogs of the genus Rana are supposed to represent the latter, but the origin of the three species occurring on Taiwan, $R$. longicrus Stejneger, 1898, R. sauteri Boulenger, 1909, and R. multidenticulata Chou and Lin, 1997 remains unclear. Of these, $R$. longicrus was previously often synonymized with the Japanese brown frog, R. japonica Günther, 1858 (Pope, 1931; Liu and Hu, 1961). Because these two species actually have a high degree of genetic compatibility (Kuramoto, 1974), $R$. longicrus is supposed to be closely related to $R$. japonica.

\footnotetext{
* Corresponding author: Tel. +81-11-706-3580;

FAX. +81-11-726-3476;
}

at Center of Experimental Plants and Animals, Hokkaido University, N10 W8 Kita-ku, Sapporo 060-0810, Japan.
On the other hand, $R$. sauteri is noted for its unique breeding ecology and larval morphology, and is occasionally assigned to a subgenus or even a genus distinct from Rana by some authors (Fei et al., 1990; Dubois, 1992). Because frogs regarded as subspecies of $R$. sauteri or species related to $R$. sauteri are distributed from southeastern China to northern Indochina (Smith, 1921, 1924; Tian and Jian, 1986; Zhao and Adler, 1993), $R$. sauteri may represent a lineage distinct from Palearctic brown frogs, and its origin is of great zoogeographical interests (Boulenger, 1909). Population systematics of $R$. sauteri (sensu lato) in Taiwan was recently revised, in which the frogs from the middle and eastern parts of the central mountain range was described as a distinct species $R$. multidenticulata, and thus $R$. sauteri (sensu stricto) was confined to populations in western lowlands (Chou and Lin, 1997b). These two species, however, have a wide intergradation zone along the western side of the central range, and are difficult to discriminate on the basis of adult morphology (Chou and Lin, 1997a, b). Therefore, in the following, these species are collectively referred as the $R$. sauteri complex.

In this paper, phylogenetic relationships among the Tai- 
wanese brown frogs and six representatives of the Japanese brown frog species ( $R$. pirica Matsui, 1991; $R$. ornativentris Werner, 1903; R. japonica, R. tsushimensis Stejneger, 1907; $R$. okinavana Boettger, 1895; and $R$. tagoi Okada, 1928) are inferred by comparing their nucleotide sequences of mitochondrial DNA (mtDNA), so as to revise the hypothesis regarding the origin of those Taiwanese species.

\section{MATERIALS AND METHODS}

DNA was extracted from small amounts (less than $50 \mathrm{mg}$ ) of frozen liver, muscle, heart, or eggs from 47 individuals representing one species and one species complex of Taiwanese and six species of Japanese brown frogs, and an outgroup taxon, $R$. catesbeiana Shaw, 1802. Locality information for these samples are given in Appendix. The published sequence of Xenopus laevis (Daudin, 1802) (Dunon-Bluteau et al., 1985) was also incorporated into the analysis as that for another outgroup.

The methods used to extract, amplify, and sequence are as described previously (Tanaka et al., 1994, 1996). A part of the mtDNA cytochrome $b$ gene was amplified using the polymerase chain reaction (PCR) and approximately 600 base-pairs (bp) of this gene were sequenced. Primers newly designated for amplification and sequencing are: L14731 (5'-GAAAAACTATCGTTGTTATTCAACTA-3'), L14850 (5'-TCTCATCCTGATGAAACTTTGGCTC-3'), H15502 (5'GGATTAGCTGGTGTGAAATTGTCTGGG-3'), and H15584 (5'CCTAGTTTATTAGGGATGGAGCGGAG-3'). Sequence numbering system followed that of the human sequence (Anderson et al., 1981).

Three different methods were employed to infer relationships among taxa on the basis of sequence data obtained; the neighborjoining method (Saitou and Nei, 1987), using NJ procedure included in the PHYLIP package (Felsenstein, 1993), the maximum likelihood method using DNAML procedure of PHYLIP, and the parsimony method using the heuristic search algorithm in PAUP (Swofford, 1993) with sequences added in a random order for 1000 repetitions. When a taxon showed some variation in the nucleotide sequence, most frequently appearing sequence was considered as the representative sequence for the taxon.

\section{RESULTS}

Genetic differentiation among taxa was estimated using nucleotide sequence data of 587 bp that were constantly obtained for all samples (Fig. 1). Intrapopulation nucleotide sequence similarities of the Rana sauteri complex were $99.8 \%$, and $99.7 \%$ for Sanyi and Wulai samples, respectively. Between samples from Alishan and Sanyi, similarities were fairly high, ranging from 95.7-95.9\%. By contrast, samples from Wulai showed a substantial sequence divergence from the above two samples with only $83.7-84.7 \%$ similarities.

In the neighbor-joining tree rooted at the midpoint of the longest path (Fig. 2A), the outgroup taxa $X$. laevis and $R$. catesbeiana were separated from the remaining frogs in order. The cluster of the ingroup, supported in $99.8 \%$ bootstrap iterations, showed nearly polytomous relationships of four major clusters.

The first cluster contained only $R$. tagoi, and the second cluster, supported by $100 \%$ iterations, consisted of two populations of the $R$. sauteri complex (Alishan and Sanyi). Sister relationship of the two individuals from Sanyi was supported by $83.4 \%$ iterations. The bootstrap value to support the mono- phyly of the first and second clusters was as low as $49.6 \%$.

The third cluster, supported by $86.8 \%$ iterations, contained $R$. okinavana, $R$. tsushimensis and the $R$. sauteri complex from Wulai. Within this cluster, $R$. okinavana first split from a subcluster consisting of the remainder, which was supported by $100 \%$ iterations. This subcluster was trichotomous with sister relationship of the two individuals of the $R$. sauteri complex from Wulai being supported only in $42.0 \%$ bootstrap iterations. This result derives from surprisingly high genetic similarities between these two individuals and $R$. tsushimensis (98.6-99.5\%).

The fourth cluster, supported by less iteration value (63.2\% iterations), consisted of $R$. longicrus, $R$. japonica, and two species with $2 \mathrm{n}=24$ chromosomes (i.e., $R$. pirica and $R$. ornativentris). The sister relationship of $R$. longicrus and $R$. japonica was only weakly supported by $47.7 \%$ iterations, while that of the two species with $2 \mathrm{n}=24$ chromosomes was supported by no less than $97.9 \%$ iterations.

The maximum-likelihood analysis, using Xenopus and $R$. catesbeiana as outgroup taxa, produced a topology identical to that obtained by neighbor-joining method (data not shown). The tree contained several collapsing branches, and confirmed the polytomous relationships of the four clusters mentioned above.

In the maximum-parsimony analysis, the sister relationship of the $R$. sauteri complex from Alishan and Sanyi and $R$. tagoi was again not supported (iterations $=38.3 \%$ ) as in the results of the above two analyses. Monophyly of $R$. japonica and $R$. longicrus, or that of these two species and the two species with $2 \mathrm{n}=24$ chromosomes was not supported, either (Fig. 2B). The bootstrap value to support sister relationship of $R$. longicrus and $R$. japonica was particularly low (42.7\%).

Monophyletic relationships of (1) the $R$. sauteri complex from Wulai, $R$. tsushimensis, and $R$. okinavana, (2) the two species with $2 \mathrm{n}=24$ chromosomes ( $R$. pirica and $R$. ornativentris), and (3) the $R$. sauteri complex from Sanyi and from Alishan were again strongly supported $(72.8 \%, 90.0 \%$ and $100 \%$ of 1,000 bootstrap iterations, respectively).

In this way, the most important point here clarified is that there are two genetically highly diverged groups within the $R$. sauteri complex; one from Alishan and Sanyi that were distinct from all other brown frogs examined here, and the other from Wulai that was close to $R$. okinavana and $R$. tsushimensis.

\section{DISCUSSION}

Rana longicrus from Taiwan, together with some brown frogs widely spread in China (e.g., R. omeimontis Ye et Fei, 1993 and $R$. zhenhaiensis Ye, Fei, et Matsui, 1995), was once regarded as conspecific with Japanese $R$. japonica from their close morphological similarities. Recent karyological and ecological studies also suggested their close phylogenetic relationships (Kuramoto et al., 1973, 1984). However, the clade of $R$. longicrus and $R$. japonica was only weakly supported both in neighbor-joining (bootstrap iterations $=47.7 \%$ ) and maximum-parsimony (42.7\%) trees, and the two species were 


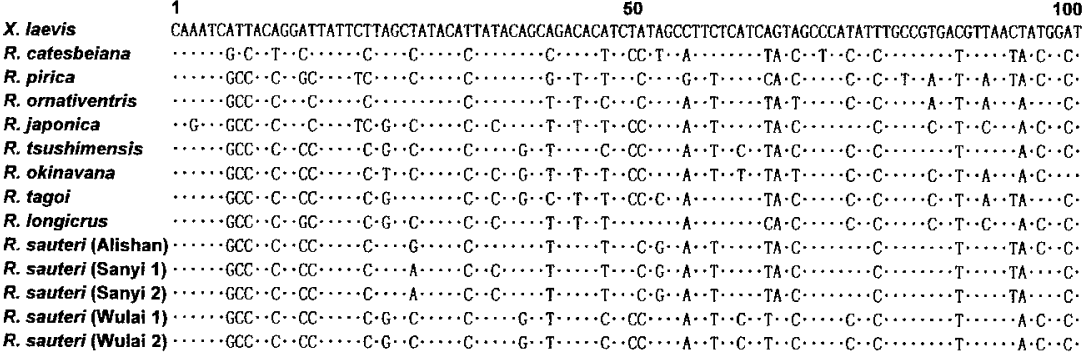

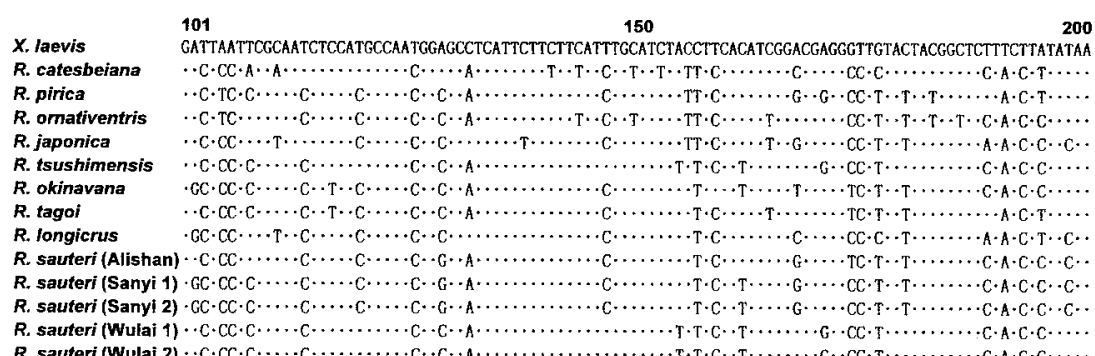

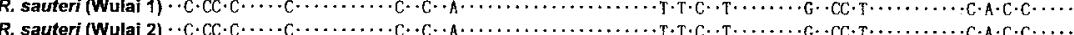

\begin{tabular}{|c|c|}
\hline & 250 \\
\hline$x$. Laevis & AGAAACATGAAATATTGGTGTGATTCTFCTATTTTTAGTTATAGCTACAGCATTTGTAGGATATGTTCTACCATGAGGACAAATATCTTTTTGAGGGGGT \\
\hline R. catesbeiana & 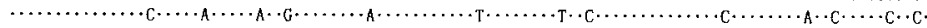 \\
\hline R. pirica & 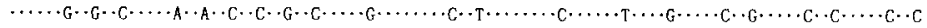 \\
\hline R. omativentris & 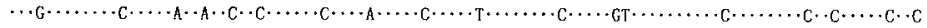 \\
\hline R. japonica & 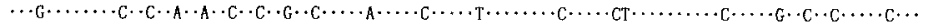 \\
\hline himensis & $\cdots \cdots G \cdot G \cdot C \cdot C \cdot G \cdot T \cdot C \cdot C \cdots C \cdot C \cdots \cdots A \cdot G \cdot C \cdot \cdots \cdots C \cdots \cdots G \cdot C \cdot C \cdot C C \cdot G \cdots \cdots G \cdot C \cdot \cdots \cdots \cdots A \cdot C \cdot \cdots \cdots C \cdot$ \\
\hline R. okinavana & 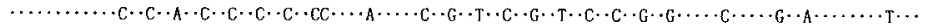 \\
\hline R. tagoi & 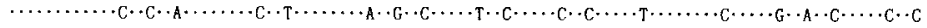 \\
\hline R. Iongicrus & 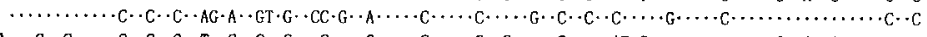 \\
\hline teri! & 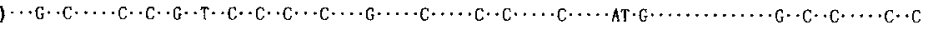 \\
\hline & 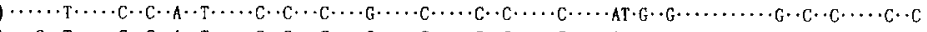 \\
\hline eri (San & $\cdot C \cdot C \cdot \cdot A \cdot+\cdots \cdots C \cdot C \cdot C \cdots C \cdots \cdot G$ \\
\hline 5 & 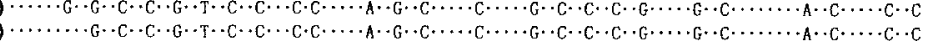 \\
\hline
\end{tabular}

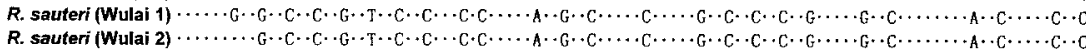

$\begin{array}{lll}301 & 350 & 400\end{array}$

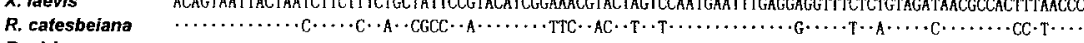

R. pirica
R.

R. ornativentris $\quad \cdots \cdots \mathrm{C} \cdot \mathrm{C} \cdot \mathrm{A} \cdots \cdots \mathrm{C} \cdot \mathrm{C} \cdot \mathrm{A} \cdot \mathrm{CGCC} \cdot \mathrm{C} \cdots \cdots \cdots \mathrm{CTTT} \cdot \mathrm{ACT} \cdots \cdot \mathrm{T} \cdots \cdots \cdot \mathrm{C} \cdot \mathrm{G} \cdots \cdots \mathrm{C} \cdots \cdots \mathrm{A} \cdots \cdots \cdots \mathrm{T} \cdot \mathrm{T} \cdot \mathrm{CC} \cdot \mathrm{C} \cdots \cdots$

R. japonica $\quad \cdots \cdots \cdots \cdot C \cdot C \cdot \cdot C \cdot C \cdot \cdot A \cdot C G C C \cdot C \cdot T \cdots \cdots C T C \cdot A C \cdot C \cdot T \cdots \cdots \cdots C \cdots \cdots \cdots C \cdot T \cdot A \cdots \cdots C \cdots \cdots A \cdot C C-C \cdots$

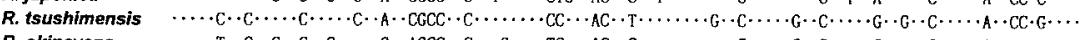

R. okinavana $\quad \cdots \cdots \mathrm{T} \cdot \mathrm{C} \cdot \mathrm{C} \cdot \mathrm{C} \cdot \mathrm{C} \cdots \cdots \mathrm{G} \cdot \mathrm{AGCC} \cdot \mathrm{C} \cdots \mathrm{G} \cdots \mathrm{TC} \cdots \mathrm{AC} \cdot \mathrm{C} \cdots \cdots \cdots \cdot \mathrm{C} \cdots \mathrm{C} \cdot \mathrm{C} \cdots \cdots \mathrm{G} \cdots \cdots \mathrm{C} \cdots \cdots \mathrm{T} \cdot \mathrm{CC} \cdot \mathrm{C} \cdot \mathrm{T}$.

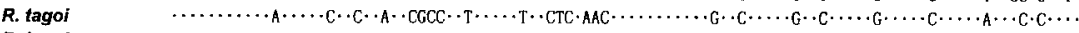

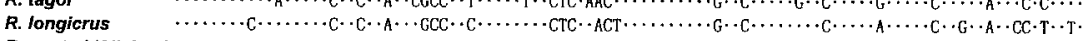

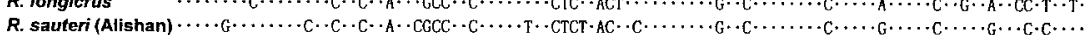

R. sauteri (Sanyi 1) $\cdots \cdots \cdots \cdots C \cdot C \cdot C \cdot C \cdot A \cdot C G C C \cdot C \cdots \cdots T \cdot C T C \cdot A A \cdot C \cdots \cdots \cdots G \cdot C \cdots \cdots G \cdot C \cdots \cdots A \cdots C \cdots \cdots A \cdots C \cdot T \cdots$

$R$, sauteri (Sanyi 2) $\cdots \cdots \cdots \cdots \cdot C \cdot C \cdot C \cdot C \cdot A \cdot C G C C \cdot C \cdots \cdots+C \cdot C T C \cdot A A C \cdot C \cdots \cdots \cdots G \cdot C \cdots G \cdot C \cdots \cdots A \cdots C \cdots \cdots A \cdots C \cdot T \cdots$

R. sauteri (Wulai 1) $\cdots \cdot C \cdot C \cdots \cdots C \cdots \cdot C \cdot A \cdot C G C C \cdot C \cdots \cdots \cdot C C \cdots A C \cdot T \cdots \cdots \cdots G \cdot C \cdots \cdots G \cdot C \cdots \cdots G \cdot G \cdot C \cdots \cdots A \cdot C C \cdot G \cdots$

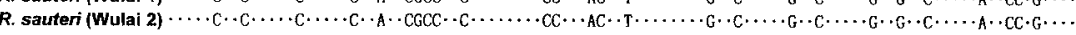

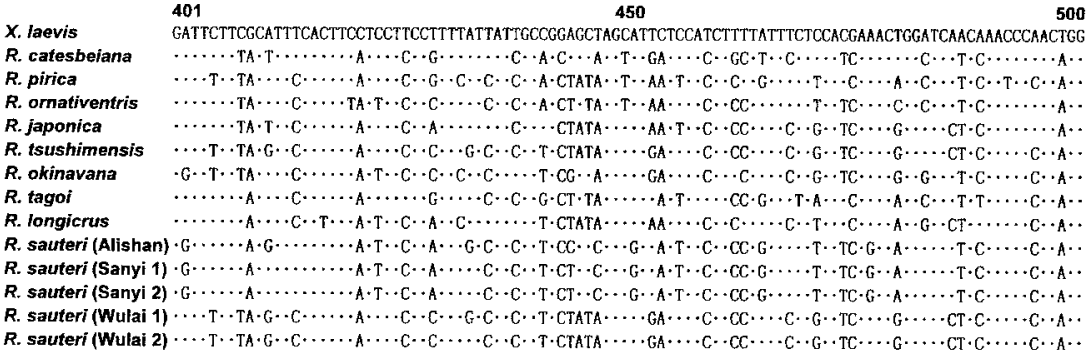

$x$. laevis 501 ATTAACTCAGACCCAGATAAAGTACCITTCCACCCATACTTCTCTTACAAAGACCTTTTAGGCTTCCTTATTATACTTACAGCACT

R. catesbeiana $\quad \mathrm{CC} \cdot \mathrm{C} \cdot \mathrm{T} \cdots \mathrm{T} \cdot \mathrm{AT} \cdots \cdots \cdot \mathrm{G} \cdot \mathrm{CT} \cdot \mathrm{C} \cdot \mathrm{T} \cdot \mathrm{T} \cdots \cdots \cdots+\mathrm{C} \cdots \cdots \cdots \cdot \mathrm{T} \cdot \mathrm{A} \cdot \mathrm{C} \cdots \cdots \cdot \mathrm{AC} \cdots \cdots \cdots \cdot \mathrm{CG} \cdots \mathrm{CT}$

R. pirica $\quad \cdot \mathrm{C} \cdot \mathrm{C} \cdots \cdots \mathrm{CA} \cdots \mathrm{T} \cdots \mathrm{C} \cdots \cdots \mathrm{CT} \cdot \mathrm{G} \cdots \cdots \cdots \cdot \mathrm{T} \cdot \mathrm{T} \cdot \mathrm{T} \cdot \mathrm{C} \cdot \mathrm{T} \cdots \cdots \cdot \mathrm{T} \cdot \mathrm{C} \cdot \mathrm{C} \cdots \cdots \cdot \mathrm{TG} \cdot \mathrm{A} \cdot \mathrm{C} \cdot \mathrm{T} \cdots \mathrm{GGT} \cdot \mathrm{C} \cdot \mathrm{C}$

$R$, ornativentris $\quad \cdot C \cdot T \cdots \cdots C A \cdot T \cdot T \cdots C \cdots \cdots C T \cdot A \cdots \cdots \cdots C \cdot \cdots \cdots \cdot C \cdots \cdots \cdots \cdot \cdot C \cdot \cdot C \cdots \cdots \cdot G \cdot A \cdot C C \cdot T \cdots G C T \cdot C \cdot$

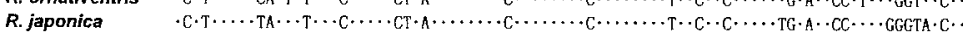

R. tsushimensis $\mathrm{TC} \cdot \mathrm{C} \cdot \mathrm{G} \cdots \mathrm{CA} \cdots \mathrm{T} \cdots \cdots \cdot \mathrm{A} \cdot \mathrm{CT} \cdot \mathrm{A} \cdots \cdots \cdot \mathrm{T} \cdots \cdots \cdots \cdot \mathrm{C} \cdots \cdots \cdots \cdots \cdot \mathrm{C} \cdot \mathrm{T} \cdots \cdots \cdot \mathrm{TG} \cdot \mathrm{GG} \cdot \mathrm{CT} \cdots \mathrm{CGGT} \cdot \mathrm{C} \cdot$

$R$, okinavana $\quad \cdot C \cdot T \cdots \cdots T A \cdots T \cdots C \cdots \cdots C \cdot A \cdots \cdots \cdot C \cdots \cdots \cdot C \cdots \cdots \cdots+C \cdot T \cdot T \cdot T G \cdot G G C C C \cdot G \cdot C G G G \cdots$

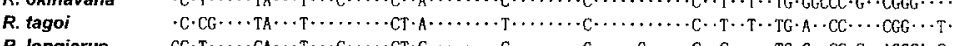

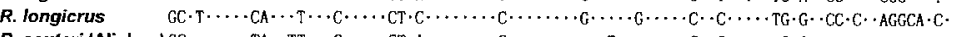

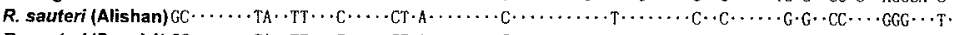

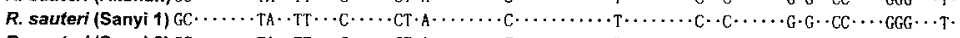

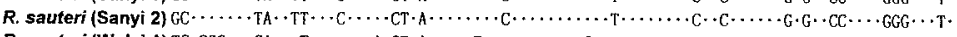

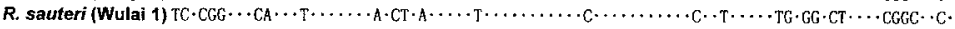

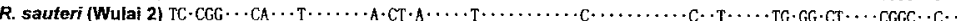

Fig. 1. Aligned sequences of a 587 bp segment of the cytochrome $b$ gene. Dots indicate identity to the sequence of Xenopus laevis. 

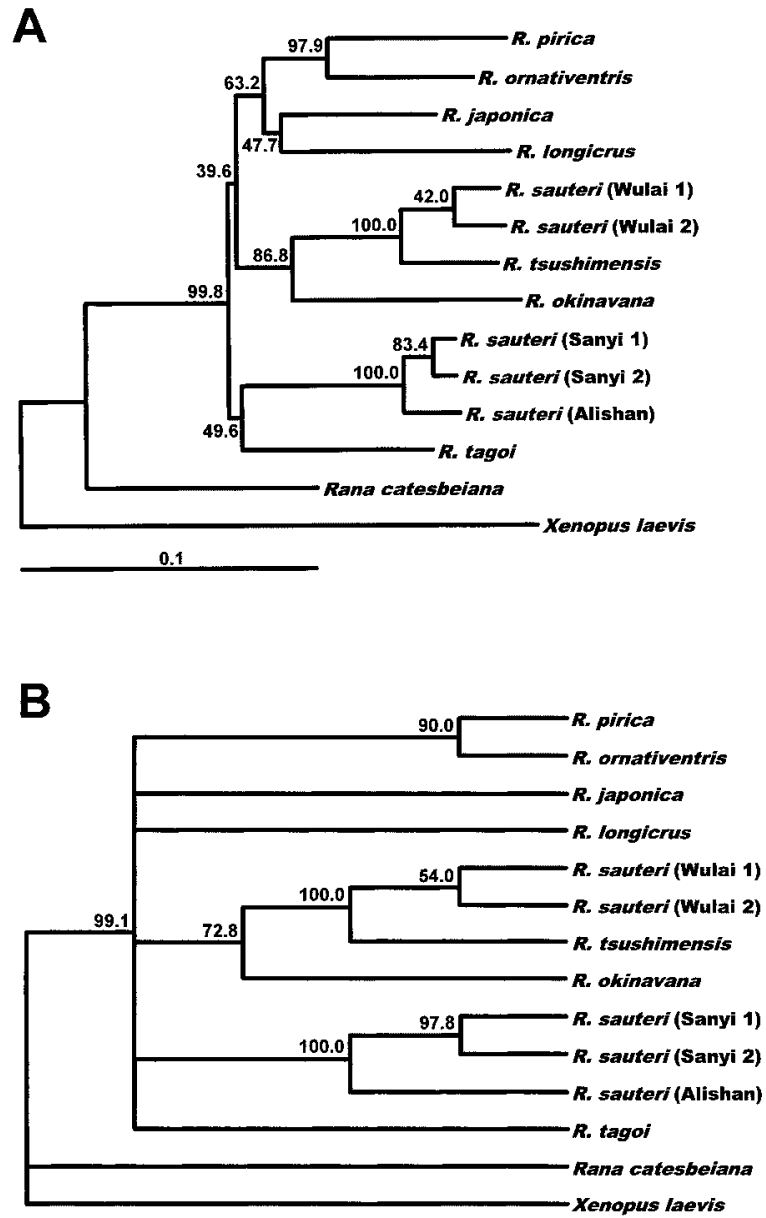

Fig. 2. A neighbor-joining tree rooted at the midpoint of the longest path (A) and a parsimony tree (B). Nodal values indicate percent support for branches in 1,000 bootstrap replicates (frequencies $<50 \%$ collapsed to polytomies in the latter tree).

suggested to be well differentiated genetically notwithstanding their close morphological and ecological similarities. These two forms have been considered distinct at the species level from the result of artificial hybridization (Kuramoto, 1974), and the present result supports this account. On the basis of genetic compatibility, Kuramoto (1974) considered that $R$. okinavana from the Ryukyus is closer to geographically remote $R$. tsushimensis from Tsushima Islands than to $R$. longicrus from Taiwan. On the other hand, from the analyses of allozymes and proteins, Nishioka et al. (1992) estimated that $R$. tsushimensis diverged much earlier than the divergence of the remaining two species. The present result greatly differs that of Nishioka et al. (1992), but almost coincides with that of Kuramoto (1974). From these results, $R$. longicrus is considered to represent a Palearctic element in Taiwan.

Our result clarified that at least two genetically distinct lineages (Alishan and Sanyi populations versus Wulai population) are included in the $R$. sauteri complex. Intrapopulation sequence similarities of this complex $(99.8 \%$ in Sanyi population and $99.7 \%$ in Wulai population) correspond to that of $R$. japonica, whereas the similarity values between Alishan and
Sanyi populations (95.7-95.9\%) are similar to those reported for local populations of $R$. tagoi from Japan (Tanaka et al., 1994). From the result of morphological analyses, Chou and Lin $(1997 \mathrm{a}, \mathrm{b})$ recently divided $R$. sauteri from Taiwan into two species and described a new species $R$. multidenticulata. These two species are reported to have a wide intergradation zone where frogs of intermediate form occur. From the distributional map they provided, Sanyi is located in the northernmost area of the range occupied by $R$. sauteri with close proximity to the intergradation zone, and Alishan and Wulai, respectively, at the central and the northernmost areas of the range of $R$. multidenticulata (Fig. 3). Diagnostic characters Chou and Lin (1997b) provided to discriminate the two species, however, are not much useful for the identification of adult specimens. Those authors assigned the population from Wulai to $R$. multidenticulata on the basis of larval morphology, but they also noted that the Wulai population is intermediate between $R$. sauteri and $R$. multidenticulata in adult morphology (Chou and Lin, 1997a). According to the present result, supposed $R$. sauteri from Sanyi and $R$. multidenticulata from Alishan are phylogenetically considerably distant from supposed $R$. multidenticulata from Wulai. This partially supports the view of Chou and Lin $(1997 a, b)$ in that it recognizes at least two distant lineages in the $R$. sauteri complex within

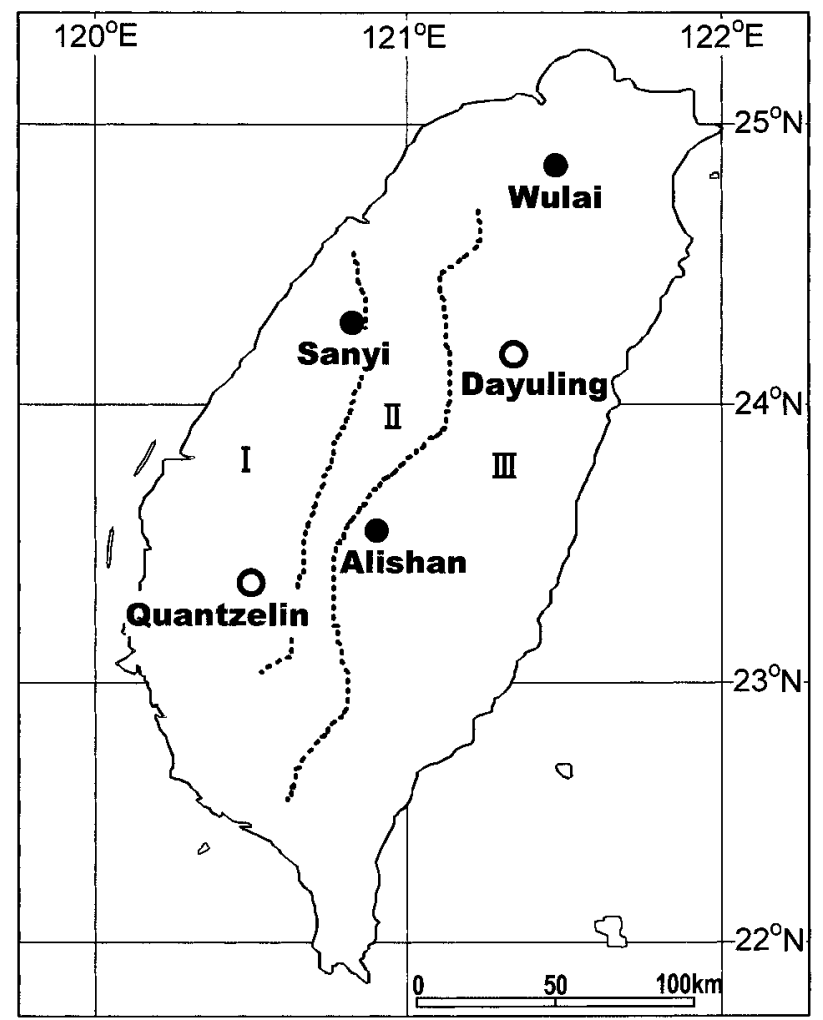

Fig. 3. A map of Taiwan showing localities where samples of the $R$. sauteri complex used in the present study were collected (closed circle) and distributional range of the $R$. sauteri complex (after Chou and Lin, 1997b: I = R. sauteri, II = intermediate, III $=R$. multidenticulata). Type localities of $R$. sauteri (Quantzelin) and $R$. multidenticulata (Dayuling) are also included (open circle). 
Taiwan. However, the contents of the two lineages are quite dissimilar between our results and Chou and Lin's (1997a, b) arrangements. In order to clarify reasons for this discordance and evaluate validity of Chou and Lin's (1997a, b) classification, whole Taiwanese populations including those from type localities of $R$. sauteri (Kanshirei = Quantzelin or Kuantzulin) and $R$. multidenticulata (Dayuling) should be surveyed in detail.

According to our result, sequence similarities of the $R$. sauteri complex from Wulai and $R$. tsushimensis, endemic to Tsushima Islands, were 98.6-99.5\%. Because these values approach to those found in a population of $R$. tsushimensis (99.5-99.8\%: Tanaka et al., 1996), the $R$. sauteri complex from Wulai is considered to be phylogenetically very close to $R$. tsushimensis. Further, these two forms are undoubtedly close to $R$. okinavana phylogenetically. Present distributional ranges of $R$. tsushimensis and $R$. okinavana are both limited to a few small islands of Japan distant from each other. From its great genetic distances from the others, $R$. okinavana is supposed to have diverged very early among these three forms. Tanaka et al. (1996) estimated that the ancestral stock of $R$. okinavana might have been isolated on the Amami and Okinawa Islands of the Central Ryukyus by the time when the Japanese main islands and the Ryukyu Archipelago were separated by the sea (Pliocene, 2-8 my B.P.: Kizaki and Oshiro, 1980). The stock one leading to $R$. okinavana and the other to the common ancestor of the remaining two forms are supposed to have diverged even earlier.

On the other hand, $R$. tsushimensis and the $R$. sauteri complex from Wulai are genetically very close and their divergence is supposed to be a rather recent event. Tsushima Islands are currently more than $1000 \mathrm{~km}$ distant from Taiwan, but because the East China Sea separating these islands is shallow ( $<200 \mathrm{~m}$ deep), these islands may have been connected by land bridges through the continent during the Pliocene (1-2 my B. P) and middle or late Pleistocene (0.020.4 my B.P.: Kimura, 1996). If this is actually the case, the ancestral stock common to the two forms would have had a wide range of distribution until rather recently. Through the subsequent sea expansion, however, the stock should have been confined to the regions corresponding to the present Tsushima Islands and Taiwan, where it has been differentiated into $R$. tsushimensis and the Wulai lineage of the $R$. sauteri complex, respectively.

The clade represented by the $R$. sauteri complex from Alishan and Sanyi is fairly differentiated genetically among the brown frogs examined here, and this lineage is supposed to have diverged at a very early stage of divergence of the East Asian brown frogs. Although the $R$. sauteri complex and $R$. tagoi are dissimilar in larval morphology and ecology, they share unique breeding ecology and egg morphology that are not found in other brown frog species (Kuramoto et al., 1984). Like the $R$. sauteri complex from Alishan and Sanyi, R. tagoi is also supposed to have diverged fairly early from other brown frogs. Each of these lines of divergence might have related to invasions into new breeding environments that were different from those utilized by most other brown frogs. Notwithstanding their genetically remote relationships, populations of the $R$. sauteri complex from Alishan and Sanyi are not easily differentiated from the population from Wulai in adult morphology. They are almost identical in the mode of larval life as well. If the present result reflects their true phylogeny, these morphological and ecological similarities are most likely the consequence of convergent evolution. Further, both of these two lineages of the $R$. sauteri complex are considered to represent not Oriental but Palearctic elements of the Taiwanese fauna like $R$. longicrus (see above).

According to the classification of Dubois (1992), which was made mainly on the morphological ground, $R$. japonica and $R$. longicrus are grouped into the $R$. japonica group. Our result, however, only weakly supported the monophyly of these two species. Because Dubois' (1992) R. japonica group also includes brown frogs from Sakhalin and the eastern Eurasian continent (Russia, Mongolia, China) as represented by $R$. amurensis, further studies including these forms are necessary to evaluate his classification on the more comprehensive phylogenetic ground.

Fei et al. (1990) split $R$. sauteri and its allies from other brown frogs as a distinct genus Pseudorana on the basis of their unique larval morphology. Dubois (1992), while largely following this idea, relegated Pseudorana to a subgenus of the genus Rana. Similar idea to regard $R$. sauteri (sensu lato) as representing a remote relationship from other brown frogs can be traced back to the original description of this species by Boulenger (1909) who suggested $R$. sauteri to bridge brown frogs and hylaranine frogs. However, our results indicate that $R$. sauteri and other brown frogs actually constitute a monophyletic group [strongly supported in both neighbor-joining (99.8\%) and maximum-parsimony (99.1\%) trees], and that the two different lineages of the $R$. sauteri complex have closer genetic relationships with different Japanese brown frogs. From such inferred phylogeny, it seems at present better not to assign the $R$. sauteri complex to a distinct genus or even subgenus but to place it in the subgenus Rana following the traditional taxonomy (e.g., Frost, 1985). Fei et al.'s (1990) genus or Dubois' (1992) subgenus Pseudorana includes several brown frogs from China and Indochina that are regarded as subspecies or relatives of $R$. sauteri (Smith, 1921; Tian and Jian, 1986; Fei et al., 1990; Zhao and Adler, 1993). In order to evaluate the validity of Pseudorana, further studies of these frogs are inevitable.

\section{ACKNOWLEDGMENTS}

We thank anonymous reviewers for helpful comments on an earlier version of this manuscript. For support in collecting sample materials we thank S. Iwanaga, Y. Misawa, T. Sato, T. Sugahara, S. Takenaka, S. Tanabe, M. Toda, and M. Toyama. This research was supported by a Grant-in-Aid from the Ministry of Education, Science, Sports and Culture of Japan (No. 07454234) and a grant from the U. S. National Geographic Society (No. 4505-91) to MM, the Research Fellowships of the Japan Society for the Promotion of Science for Young Scientists (No. 6050) and by the Sasakawa Scientific Research Grant from the Japan Science Society to TT-U, and a Grant for For- 
eign Visiting Researchers from the Taiwan Museum to $\mathrm{HO}$.

\section{REFERENCES}

Anderson S, Bankier AT, Barrell BG, de Bruijin MHL, Coulson AR, Drouin J, Eperon IC, Nierlich DP, Roe BA, Sanger F, Schreier $\mathrm{PH}$, Smith AJH, Staden R, Young IG (1981) Sequence and organization of the human mitochondrial genome. Nature 290: 457465

Boulenger GA (1909) Descriptions of four new frogs and a new snake discovered by Mr. H. Sauter in Formosa. Ann Mag Nat Hist 8: 492-495

Chou WH, Lin JY (1997a) Geographical variations of Rana sauteri (Anura: Ranidae) in Taiwan. Zool Stud 36: 201-221

Chou WH, Lin JY (1997b) Description of a new species, Rana multidenticulata (Anura: Ranidae), from Taiwan. Zool Stud 36: 222-229

Dubois A (1992) Notes sur la classification des Ranidae (Amphibians Anoures). Bull Mens Soc Linn Lyon 61: 350-352

Dunon-Bluteau D, Volovitch M, Brun G (1985) Nucleotide sequence of a Xenopus laevis mitochondrial DNA fragment containing the $D$-loop, flanking tRNA genes and the apocytochrome $b$ gene. Gene 36: 65-78

Fei L, Ye CY, and Huang YZ (1990) Key to Chinese Amphibia. Chongqing Branch, Sci Technol Lit Press, Chongqing

Felsenstein J (1993) PHYLIP (phylogeny inference package) Version 3.5c. Distributed by the author. Dept Genet Univ Washington, Seattle

Frost DR (1985) Amphibian Species of the World: A Taxonomic and Geographical Reference. Allen Press, Lawrence

Kimura M (1996) Paleogeography of the Ryukyu Arch. Chikyu Monthly 18: $488-494$

Kizaki K, Oshiro I (1980) The origin of the Ryukyu Islands. In "Natural History of Ryukyu" Ed by K Kizaki, Tsukiji-shokan, Tokyo, pp 837

Kuramoto M (1974) Experimental hybridization between the brown frogs of Taiwan, the Ryukyu Islands and Japan. Copeia 1974: 815-822

Kuramoto M, Furuya E, Takegami M, Yano K (1973) Karyotypes of the six species of frogs from Japan and Taiwan. Bull Fukuoka Univ Educ Pt III 23: 67-78

Kuramoto M, Wang CS, Yu HT (1984) Breeding, larval morphology and experimental hybridization of Taiwanese brown frogs, Rana longicrus and $R$. sauteri. J Herpetol 18: 387-395

Liu CC, Hu SC (1961) Chinese Tailless Batrachians. Science Press, Beijing

Nishioka M, Sumida S, Borkin LJ, Wu ZA (1992) Genetic differentiation of 30 populations of 12 brown frog species distributed in the Palearctic region elucidated by the electrophoretic method. Sci
Rep Lab Amphib Biol Hiroshima Univ 11: 109-160

Pope CH (1931) Notes on amphibians from Fukien, Hainan, and other parts of China. Bull Amer Mus Nat Hist 61: 397-611

Saitou N, Nei M (1987) The neighbor-joining method: a new method for reconstructing phylogenetic trees. Molec Biol Evol 4: 406425

Smith MA (1921) New or little-known reptiles and batrachians from Southern Annan (Indo-China). Proc Zool Soc Lond 1921: 423440

Smith MA (1924) Descriptions of Indian and Indo-Chinese tadpoles. Rec Indian Mus 26: 137-143

Swofford DL (1993) Users Manual for PAUP Version 3.1: Phylogenetic analysis using parsimony. Illinois Nat Hist Survey, Champaign, Illinois

Tanaka T, Matsui M, Takenaka O (1994) Estimation of phylogenetic relationships among Japanese brown frogs from mitochondrial Cytochrome b gene (Amphibia: Anura). Zool Sci 11: 753-757

Tanaka T, Matsui M, Takenaka O (1996) Phylogenetic relationships of Japanese brown frogs (Rana: Ranidae) assessed by mitochondrial cytochrome b gene sequences. Biochem Syst Ecol 24: 299-307

Tian WS, Jian YM (1986) Identification Manual for Chinese Amphibians and Reptiles. Science Press, Beijing

Zhao EM, Adler K (1993) Herpetology of China. Contrib Herpetol 10: $1-522$

\section{APPENDIX}

Material Examined: Sources of tissue samples used are as follows. A total of 47 frogs are stored at the Graduate School of Human and Environmental Studies, Kyoto University (KUHE). Rana catesbeiana $(n=4)$ : Inuyama-shi, Aichi $(n=2)$; Bungotakada-shi, Oita $(n=2)$. Rana pirica $(n=5)$ : Obihiro-shi, Hokkaido $(n=1)$; Fukushima-cho, Hokkaido $(n=1)$; Sapporo-shi, Hokkaido $(n=3)$. Rana ornativentris $(n=6)$ : Towadako-machi, Aomori $(n=1)$; Oyamamachi, Toyama $(n=2)$; Sasayama-cho, Hyogo $(n=1)$; Tosayamamura, Kochi $(n=1)$; Bungotakada-shi, Oita $(n=1)$. Rana japonica ( $n$ = 15): Tateyama-shi, Chiba $(n=12)$; Togane-shi, Chiba $(n=1)$; Maizuru-shi, Kyoto $(n=1)$; Bungotakada-shi, Oita $(n=1)$. Rana tsushimensis $(\mathrm{n}=3)$ : Mitsushima-cho (Tsushima Isl.), Nagasaki $(\mathrm{n}=$ 3). Rana okinavana $(n=1)$ : Higashi-son, Okinawa $(n=1)$. Rana tagoi $(n=7)$ : Towadako-machi, Aomori $(n=1)$; Hayakawa-cho, Yamanashi $(n=1)$; Kyoto-shi, Kyoto $(n=3)$; Tosayama-mura, Kochi $(n=1)$; Gokase-cho, Miyazaki $(n=1)$. Rana longicrus $(n=1)$ : Keelung, Taipei $(\mathrm{n}=1)$. Rana sauteri complex $(\mathrm{n}=5)$ : Alishan, Chiayi $(=R$. multidenticulata, $\mathrm{n}=1)$; Sanyi, Miaoli $(=R$. sauteri, $\mathrm{n}=2)$; Wulai, Taipei (= $R$. multidenticulata, $\mathrm{n}=2$ ).

(Received November 27, 1997 / Accepted January 19, 1998) 